\title{
Situs inversus in a Dog
}

\section{Renata Meira Lopes de Castro Mello', Flávia Bormann Vieira Nassif², Flávio Guilherme Costa Lima', Marjane Santos de Matos Carvalho ${ }^{3}$ \& Renata Santiago Alberto Carlos ${ }^{4}$}

\begin{abstract}
Background: Situs inversus (SI) is a rare congenital malformation characterized by the transposition of the viscera (thoracic and/or abdominal) to the opposite side of its normal topography, as a mirror image. In situs inversus totalis (SIT), all organs are inverted, in addition to the cardiac apex being directed to the right side of the body (dextrocardia). It may not present any clinical signs, and hence, it may be an accidental finding. Because of the rare occurrence of SIT, no epidemiological studies have been conducted in dogs. In 50\% of the cases, it may be associated with primary ciliary dyskinesia (PCD), which causes respiratory clinical signs. SIT can be diagnosed using routine examinations such as ultrasonography, radiography, tomography, and echocardiography. The objective of this paper was to describe the radiographic and echocardiographic findings in a dog with SIT.

Case: A 4-month-old Yorkshire Terrier bitch was admitted to the veterinary clinic, with a clinical complaint of cough. A physical examination showed that the dog's general conditions were good; it had normal appetite and the mucous membranes had a normal color. Radiographic examination revealed changes in the topographic anatomy of the organs. The cardiac silhouette was inverted, adopting a " $\mathrm{D}$ " form; this suggested that the left-side chambers were positioned on the right side, and the right-side chambers were located in the left topographic region. The aortic arch was visible on the right side of the thorax. A diffuse non-structured pulmonary interstitial pattern was observed on latero-lateral projections, which could be associated with an inflammatory process. The fundic region of the stomach, spleen, and thymus were visible on the right side. The liver was on the left side of the patient's abdomen. The stomach contained a foreign body of radiopaque nature that was considered a radiographic finding. Echocardiography revealed the right atrium on the left, and the left atrium on the right side. The diagnosis was SIT. The prescribed treatment included prednisolone $(1 \mathrm{mg} / \mathrm{Kg}$ SID) and $N$ acetylcysteine (10 mg/kg BID), both for 7 days, based on the possibility of bronchitis. Upon return after medication, the animal showed no clinical signs of cough. After 1 year of SIT diagnosis, the animal has remained asymptomatic and has shown good physical development.

Discussion: Dogs with SIT do not usually show clinical signs, and the condition is considered an accidental finding on complementary examinations such as ultrasonography, radiography, tomography, and echocardiography. The radiographic examination associated with echocardiography allowed the identification of SIT, which in this case was not associated with PCD because the respiratory clinical signs had not reappeared after treatment. The prognosis in this case was considered good, corroborating the findings of previous studies showing that the prognosis of individuals with isolated SI is good in the absence of any structural cardiac alteration or other underlying diagnoses, with the average life expectancy being similar to that of the general population. In conclusion, when isolated, SIT is an abnormality that is difficult to diagnose because it does not necessarily present clinical signs. The case reported here contributes to the literature because it documents an accidental finding of an isolated case of SIT in a young dog, which had a good prognosis and quality of life after treatment.
\end{abstract}

Keywords: dog, ciliary disease, inversion of organs. 


\section{INTRODUCTION}

Situs inversus (SI) is a rare congenital malformation characterized by the transposition of the viscera to the opposite side of its normal topography, as a mirror image $[1,18]$. In situs inversus totalis (SIT), all organs are inverted, in addition to the cardiac apex being directed to the right side of the body [15].

SI has been reported in association with primary ciliary dyskinesia (PCD) in some dogs. PCD is a rare autosomal recessive disorder characterized by abnormality in ciliary functions, causing abnormal mucociliary clearance $[5,16]$. The cilia of the nodal cells and the notochordal plate of the embryo shows a certain direction and position of beats, determining the laterality of the viscera. However, when there is ciliary immobility, visceral symmetry is randomized, and the association between PCD and SI occurs [3,20].

Owing to the rarity of SIT, no epidemiological studies have been conducted on dogs [7]. In the abscence of clinical signs, SI is usually an accidental finding [17]. Some dog breeds have been reported in the literature to develop SI such as English Cocker Spaniel, Dalmatian, Golden Retriever, Pekingese, Dachshund, Old English Sheepdog, Shih Tzu and Chow-chow $[4,6,9,13,14,17,19]$.

SIT can be diagnosed using routine examinations such as ultrasonography, radiography, tomography, and echocardiography $[8,17]$. In cases in which SIT is associated with PCD, the therapeutic approach is to manage the changes resulting from PCD, while avoiding obesity, exposure to respiratory epithelial aggressors, and excessive excitement [10]. The objective of this paper was to describe the radiographic and echocardiographic findings in a dog with SIT.

\section{CASE}

A 4-month-old Yorkshire Terrier bitch was admitted to the veterinary clinic with a complaint of cough. A physical examination showed that the dog's general conditions were good; it had normal appetite and the mucous membranes had a normal color.

A respiratory investigation using chest radiography was performed in the dorsal ventral position, and it revealed the reversal of the cardiac silhouette, which adopted a "D" form. This suggested that the left-side chambers were positioned on the right side, and the right-side chambers were located in the left topographic region. The aortic arch and thymus were both visible on the right side of the thorax (Figure 1). A diffuse non-structured pulmonary interstitial pattern was observed on latero-lateral projections, which could be associated with an inflammatory process (Figure 2).

Owing to the size of the patient, the abdominal structures were visualized by radiography: the fundic region of the stomach and spleen were visible on the right side. The liver was on the left side of the patient's abdomen. The stomach contained a foreign body of radiopaque nature that was considered a radiographic finding because of the absence of clinical signs compatible with it (Figure 1).

Echocardiography revealed the inversion of the cardiac chambers (Figure 3). After analyzing all the imaging findings, the dog was diagnosed with SIT.

The prescribed treatment included prednisolone $\left(\text { Prediderm }{ }^{\circledR}\right)^{1}[1 \mathrm{mg} / \mathrm{kg} \mathrm{SID}]$ and $\mathrm{N}$-acetylcysteine (Mucomucil syrup $\left.^{\circledR}\right)^{2}$ [10 mg/kg BID], both for 7 days, based on the possibility of allergic bronchitis. Upon return after medication, the animal showed no clinical signs of cough. After 1 year of SIT diagnosis, the animal has remained asymptomatic and has shown good physical development.

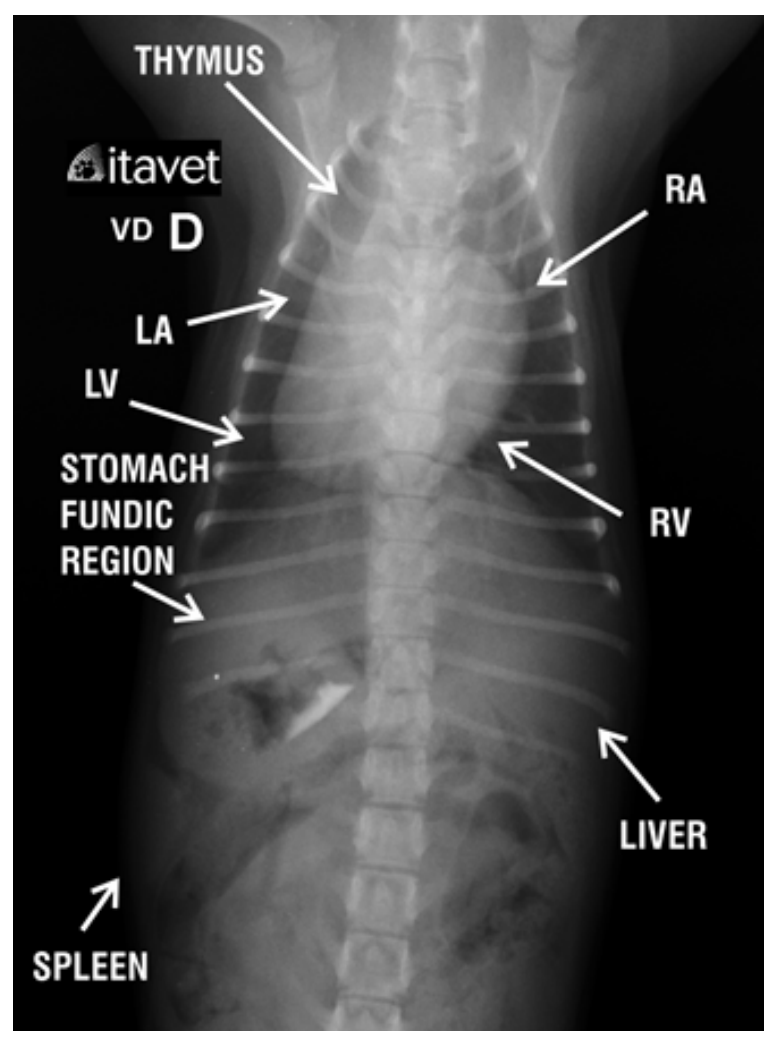

Figure 1. Inversion of the cardiac silhouette, with the left-side chambers positioned to the right side (LA, LV), and the right-side chambers located on the left side (RA, RV); the fundic region of the stomach, spleen, and thymus are visible on the right side; and the liver is visible on the left side. Legend: Thymus; Left atrium (LA); Left ventricle (LV); Stomach, fundic region; Spleen; Liver; Right ventricle (RV); and Right atrium (RA). 


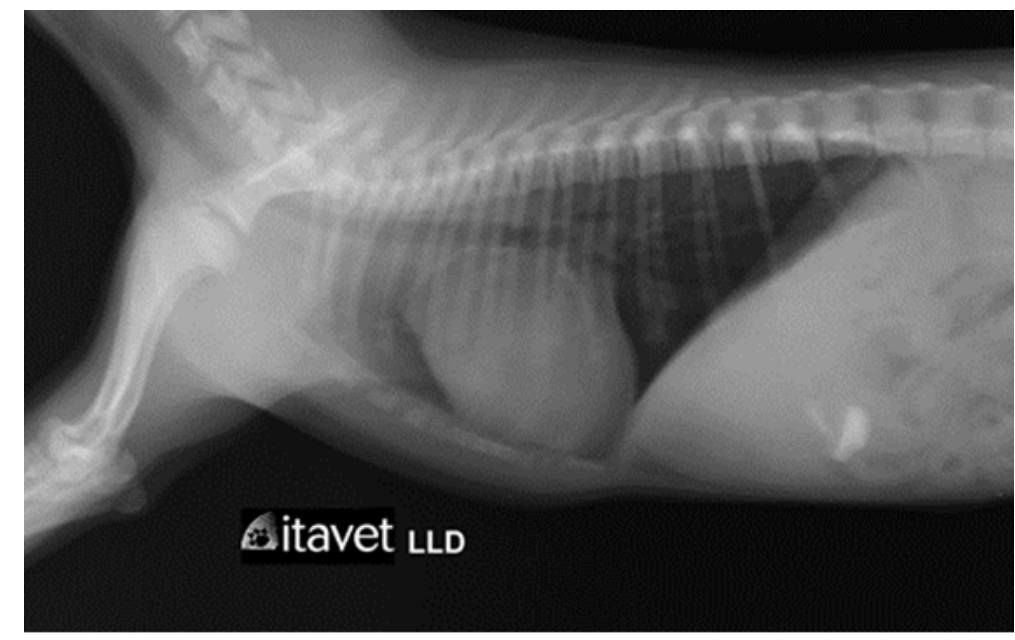

Figure 2. Radiographic image of the right latero-lateral projection, showing the presence of a diffuse non-structured pulmonary interstitial pattern.

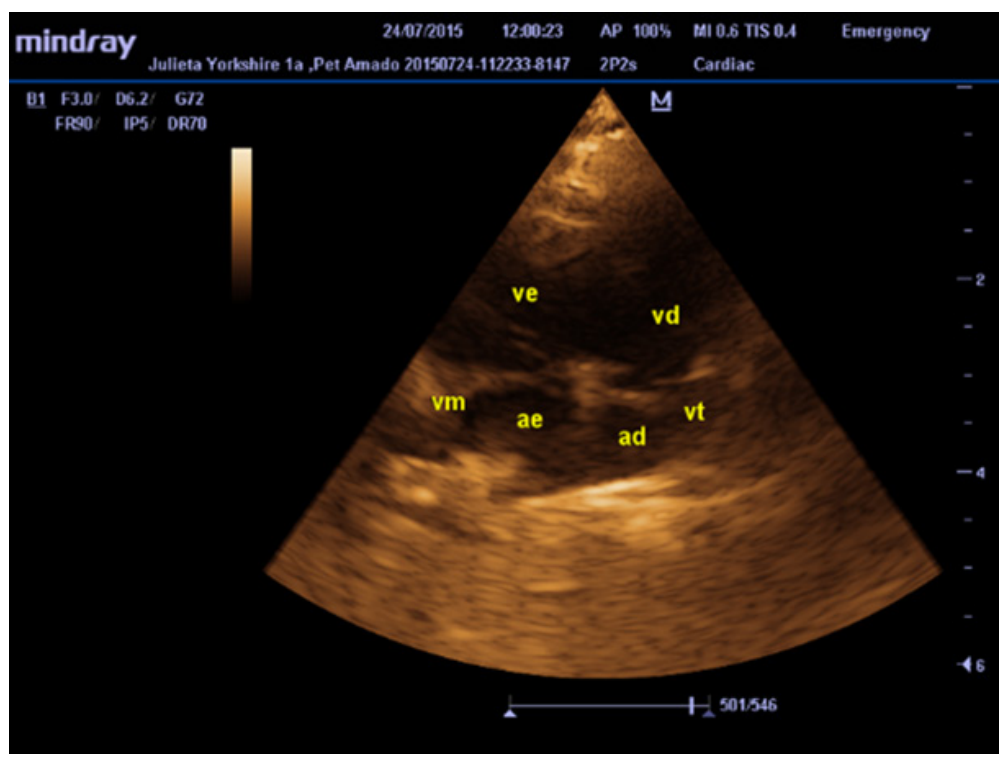

Figure 3. Echocardiogram showing the inversion in the positions of the cardiac chambers: left-side chambers (LV, LA, MV) are positioned on the right side; right-side chambers (RV, RA, TV) are positioned on the left side of the thorax. Legend: Left ventricle (ve); Mitral valve (va); Left atrium (ae); Right ventricle (vd); Tricuspid valve (vt); and Right atrium (ad).

\section{DISCUSSION}

The bitch in the referred case presented with cough as the main clinical complaint. A physical examination showed that its general conditions were good. The inversion of organs may not present clinical signs, and can therefore be an accidental finding on imaging studies [12].

The definitive diagnosis of SIT in the referred patient was made through radiographic and echocardiographic examinations. This was consistent with reports in the literature, which reported that ultrasonography, radiography, tomography, and echocardiography are necessary for the definitive diagnosis of SIT $[8,17]$.

An association between SIT and PCD was initially suspected in this patient because of the presence of respiratory signs. According to the literature, the clinical signs associated with PCD can range from recurrent or persistent respiratory infections [21] to hydrocephalus, otitis media with hearing loss, intolerance to exercise, male infertility, and female subfertility $[13,17]$. 
As a result of the treatment with corticosteroids and antibiotics, the patient showed a clinical improvement within 7 days, and because no respiratory clinical signs reappeared within 1 year of follow-up, the possibility of the association with PCD was discarded. According to the literature, individuals with PCD have a $50 \%$ chance of developing SI because the functioning of the cilia normally determines the position of the internal organs during early embryonic development $[2,8,13,16]$.

The prognosis in this case was considered good. This corroborated with the findings of previous studies showing that the prognosis of individuals with isolated SI is good in the absence of any structural cardiac alteration or other underlying diagnoses, with the average life expectancy being similar to that of the general population [11].
In conclusion, when isolated, SIT is an abnormality that is difficult to diagnose because it does not necessarily present clinical signs. The case reported here contributes to the literature because it documents an accidental finding of an isolated case of SIT in a young dog, which had a good prognosis and quality of life after treatment.

\section{MANUFACTURERS}

${ }^{1}$ Ouro Fino Saúde Animal Ltda. Cravinhos, SP, Brazil.

${ }^{2}$ Vetnil Indústria e Comércio de Produtos Veterinários Ltda. Louveira, SP, Brazil.

Acknowledgments. The authors would like to thank UESC for their support in the publication of this article.

Declaration of interest. The authors report that there are no conflicts of interest in the present work. The authors are solely responsible for the content and writing of the article.

\section{REFERENCES}

1 Adams R. \& Churchill E.D. 1937. Situs inversus, sinusitis, bronchiectasis. A report of five cases, including frequency statistics. The Journal of Thoracic and Cardiovascular Surgery. 7: 206-217.

2 Afzelius B.A. 1998. Immotile cilia syndrome: past, present and prospects for the future. Thorax. 53(10): 894-897.

3 Afzelius B.A. 1999. Asymmetry of cilia and of mice and men. The International Journal of Developmental Biology. 43(4): 283-286.

4 Cahua J., Dias D. \& Vieira O.G. 2015. Complete Situs Inversus in 2 Asymptomatic Dogs. Topics in Companion Animal Medicine. 30(2): 68-71.

5 Carlén B. \& Stenram U. 2005. Primary ciliary dyskinesia: a review. Ultrastructural Pathology. 29: 217-220.

6 Cavrenne R., Busscher V., Bolen G., Billen F., Clercx C. \& Snaps F. 2008. Primary ciliary dyskinesia and situs inversus in a young dog. Veterinary Record. 163(2): 54-55.

7 Beths T., McEntee K., Votion D., Snaps F., Henroteaux M. \& Jorissen M. 2000. Use of cilia genesis in the diagnosis of primary ciliary dyskinesia in a dog. Journal of the American Veterinary Medical Association. 217(11): 1681-1689.

8 Durant A.M. 2008. What is your diagnosis? Thoracic and abdominal situs inversus totalis. Journal of the American Veterinary Medical Association. 232(2): 197-198.

9 Edwards D.F., Kennedy J.R., Toal R.L., Maddux J.M., Barnhill M.A. \& Daniel G.B. 1989. Kartagener's Syndrome in a Chow Chow Dog with Normal Ciliary Ultrastructure. Veterinary Pathology. 26: 338-340.

10 Hawkins E.C. 1992. Doenças respiratórias. In: Nelson R.W. \& Couto C.G. (Eds). Fundamentos de medicina interna de pequenos animais. Rio de Janeiro: Guanabara Koogan, pp.153-157.

11 Marques P., Moura C., Spratley J., Clemente L.P. \& Clemente M.P. 2006. Situs inversus na criança - Manifestações otorrinolaringológicas. Revista Portuguesa de Otorrinolaringologia e Cirurgia Cérvico-Facial. 44(4): 365-376.

12 Merveille A.C., Battaille G., Billen F., Deleuze S., Fredholm M., Thomas A., Clercx C. \& Lequarré A.S. 2014. Clinical Findings and Prevalence of the Mutation Associated with Primary Ciliary Dyskinesia in Old English Sheepdogs. Journal of Veterinary Internal Medicine. 28(3): 771-778.

13 Niel J.A., Canapp S.O., Cook C.R. \& Lattimer J.C. 2002. Kartagener's syndrome in a Dachshund dog. Journal of the American Animal Hospital Association. 38: 45-49.

14 Oui H., Kim J., Bae Y., Oh J., Park S., Lee G., Jeon S. \& Choi J. 2013. Computed Tomography Angiography of Situs Inversus, Portosystemic Shunt and Multiple Vena Cava Anomalies in a Dog. Journal of Veterinary Medical Science. 75: 1525-1528.

15 Passos M.D., Toso K.A., Miranda J.S. \& Osella O.F.S. 2009. Associação entre Dextrocardia em Situs Inversus Totalis, Comunicação Interventricular e Forma Incomum de Estenose Subaórtica Membranosa em Paciente Adulta. Revista Brasileira de Ecocardiografia e Imagem Cardiovascular. 22(4): 43-46. 
16 Piantedosi D., Cortese L., Meomartino L., Loria A.D. \& Ciaramella P. 2011. Situs inversus totalis associated with subaortic stenosis, restrictive ventricular septal defect, and tricuspid dysplasia in an adult dog. The Canadian Veterinary Journal. 52(11): 1237-1242.

17 Reichler I.M., Hoerauf A., Guscetti F., Gardelle O., Stoffel M.H., Jentsch B., Walt H. \& Arnold S. 2001. Primary ciliary dyskinesia with situs inversus totalis, hydrocephalus internus and cardiac malformations in a dog. Journal Small Animal Practice. 42: 345-348.

18 Shiraiwa K., Watanabe A., Sato N., Nito M. \& Kobayashi Y. 1995. Case report on situs inversus totalis in two Sprague-Dawley rats. Experimental Animals. 44(4): 341-345.

19 Souza Junior P., Silva S.S.R. \& Martins M.C. 2011. Síndrome de Kartagener em um cão (Canis lupus familiaris) da raça Cocker Spaniel Inglês. Arquivo Brasileiro de Medicina Veterinária e Zootecnia. 63: 768-772.

20 Waters A.M. \& Beales P.L. 2011. Ciliopathies: an expanding disease spectrum. Pediatric Nephrology. 26(7): 10391056.

21 Zariwala M.A., Knowles M.R. \& Omran H. 2007. Genetic defects in ciliary structure and function. Annual review of physiology. 69: 423-450. 